\title{
BMJ Open Patients' information needs and attitudes about post-treatment surveillance for colorectal cancer in the United States: a multi-perspective, mixed methods study
}

\author{
Lisa M Lowenstein, ${ }^{1}$ Robert J Volk, ${ }^{1}$ Amanda Cuddy, ${ }^{2}$ Andrea P Hempstead, ${ }^{1}$ \\ Y Nancy You, ${ }^{2}$ Katherine Van Loon, ${ }^{3}$ Stefanos Millas, ${ }^{4}$ Jeffrey A Meyerhardt, ${ }^{5}$ \\ Patrick Gavin, ${ }^{6}$ George J Chang ${ }^{2}$
}

To cite: Lowenstein LM Volk RJ, Cuddy A, et al. Patients' information needs and attitudes about post-treatment surveillance for colorectal cancer in the United States: a multi-perspective, mixed methods study. BMJ Open 2019;9:e025888. doi:10.1136/ bmjopen-2018-025888

- Prepublication history and additional material for this paper are available online. To view these files, please visit the journal online (http://dx.doi org/10.1136/bmjopen-2018025888).

Received 12 April 2019 Revised 03 July 2019 Accepted 19 July 2019

\section{Check for updates}

(c) Author(s) (or their employer(s)) 2019. Re-use permitted under CC BY-NC. No commercial re-use. See rights and permissions. Published by BMJ.

For numbered affiliations see end of article.

\section{Correspondence to}

Dr Lisa M Lowenstein;

LMLowenstein@mdanderson. org

\section{ABSTRACT}

Objective We sought to determine patients' informational needs for post-treatment surveillance and elicit clinicians' and patient advocates' (ie, stakeholders) opinions regarding what patients should know about post-treatment surveillance in the USA.

Design A mixed-methods study, using semi-structured interviews followed by a survey study.

Setting Participants for the interviews were from two large academic medical centres and a safety-net hospital. The stakeholders were recruited from attendees at the Alliance for Clinical Trials in Oncology Network Spring 2016 meeting.

Participants Participants for the in-depth interviews were purposively sampled. Eligible patients were 6 months to 5 years post curative resection for colorectal cancer and were fluent in English. Participants for the anonymous survey were stakeholders.

Main outcome(s) and measure(s) The main outcome was patients' with colorectal cancer informational needs for post-treatment surveillance, using an interview guide. The second outcome was the importance of the identified informational needs using an anonymous survey. Results Of the 67 patients approached, 31 were interviewed (response rate $=46 \%$ ), the majority were between 1 and 3 years post-treatment $(81 \%)$ and diagnosed at stage III (74\%). Despite a desire to monitor for cancer recurrence, patients had little understanding of the concept of post-treatment surveillance, equating surveillance with screening and a belief that if a recurrence was found early there would be a higher likelihood of cure. The survey suggested that clinicians $(n=38)$ and patient advocates $(n=11)$ had some differing opinions regarding what patients should know about surveillance to be active in decisions. For example, compared with clinicians, patient advocates felt that patients should know recurrence treatment options $(100 \%$ vs $58 \%$ ) and likelihood for cure following recurrence treatment (100\% vs $38 \%)$.

Conclusions The results of this exploratory mixedmethods study suggest that novel educational interventions targeting both patients and clinicians are
Strengths and limitations of this study

- Patients were recruited from multiple clinical settings, representing patients from different socioeconomic backgrounds.

- One of the few studies to examine patients' informational needs following curative treatment for colorectal cancer, surveillance.

- We used convenience sampling of attendees at a single meeting for the survey portion of the study.

- The sample size for the stakeholder survey was small and exploratory in nature.

needed to address the informational needs for posttreatment surveillance of colorectal cancer.

\section{BACKGROUND}

Colorectal cancer (CRC) is the third leading cancer in men and women and the fourth most common cancer overall. ${ }^{12}$ Nearly $80 \%$ of all newly diagnosed patients with CRC will be eligible for curative resection followed by post-treatment surveillance to detect recurrence and manage treatment associated effects. ${ }^{3}$ Among those patients who develop recurrence, approximately one in three to four patients will be eligible for salvage surgery with curative intent. ${ }^{4-6}$ Salvage surgery is associated with long-term survival of $30 \%-50 \%$. However, not all patients will develop recurrence or be eligible for salvage resection, and it is estimated that between 15 and 50 patients undergo repeated surveillance testing to identify one patient eligible for salvage surgery.

Current guidelines for the frequency or duration of surveillance evaluation are variable, and there is uncertainty regarding 
the optimal timing, frequency, duration and modality of surveillance monitoring that should be conducted and for whom. ${ }^{7}$ As a result, it is imperative that patients are active in their care and knowledgeable about CRC post-treatment surveillance and recurrence.

Prior studies have shown that survivors who have had curative resection for CRC have limited knowledge about surveillance testing and risks for recurrence. ${ }^{8}$ However, these previous studies provide limited guidance about identifying different key facts survivors should understand so they can be active participants in decision making about CRC post-treatment surveillance planning or recurrence treatment. The purpose of this study was to identify patients' with CRC informational needs and elicit clinicians' and patient advocates' opinions regarding what patients should know about post-treatment surveillance to promote active participation in decisions about post-treatment surveillance.

\section{METHODS}

\section{Study design}

This was a mixed-methods study consisting of semistructured, in-depth interviews with patients and a stakeholder survey with clinicians and patient advocates.

\section{Patient and public involvement}

Patient advocates, clinicians, and researchers from the Alliance for Clinical Trials in Oncology were involved in all aspects of the study from development of the research question, outcome measures, study design, and data collection strategies during our biannual meetings. We presented the results to the stakeholders at an in-person meeting. Results were not shared with patient participants.

\section{Study procedures}

\section{Patients}

Patients were recruited from two large academic cancer centres in the West and Southwest regions and one safety-net hospital in the Southwest region of the United States. Eligible patients were $\geq 18$ years of age, 6 months to 5 years from curative resection of their colon or rectum, and were fluent in English. Researchers with no prior relationship with potential participants reviewed the upcoming surveillance appointments to identify eligible patients and assess interest in participating. After consent, the interviews were conducted either by phone or in-person by a male (VFR) with over 5 years of research experience with a Bachelors degree or a female (APH) research assistant with 2-3 years of research experience with a Bachelors degree. Both were trained by the first author to conduct the interviews. Interview participants knew the interviewers name and the purpose of the study. All interviews were conducted from September 2014 to July 2016, lasted about an hour, and were audio-recorded and transcribed verbatim. Transcripts were not returned to the participants for comment and/or corrections.
Clinicians and patient advocates (stakeholders)

Stakeholders who attended the Spring 2016 meeting of the Alliance for Clinical Trials in Oncology Network were eligible to participate. The stakeholders included gastrointestinal clinicians from academic and non-academic community-based practices and patient advocates. From here on out, the term 'advocates' refers to 'patient advocates'.

\section{Data collection instruments and analysis}

\section{Semistructured interview guide and analysis}

The semistructured interview guide was developed by review of the literature and expert opinion and was iteratively refined during the data collection period. The final guide is available by request.

Framework method guided the thematic analysis. ${ }^{9}$ Two or more researchers (LML, AC, GJC) coded all transcripts and met to discuss the coded transcripts. The coded texts were labelled with both deductive and inductive codes. The deductive codes were derived from the interview guide, and the inductive codes were developed iteratively. The coded texts were grouped together into themes and subthemes to describe the range of patient knowledge and attitudes regarding CRC surveillance. The coded text was rated on a dichotomous scale or a Likert scale ranging from low knowledge to high knowledge (table 1).

The analysis also included comparing the themes and sub-themes across groups, such as comparing patients who were high versus low risk for recurrence based on stage of diagnosis and comparing patients from the different recruitment sites. Atlas.ti V.7 was used to facilitate analysis of the coded transcripts.

\section{Stakeholder survey}

Using the data from the qualitative interviews, we developed an anonymous survey to elicit stakeholders opinions regarding what patients should know about post-treatment surveillance. Additionally, candidate items were initially taken from published guidelines and expert opinion. The candidate list was reviewed by LML, AC and GJC for clarity, redundancy, and importance to determine the necessary facts and key messages to include in the survey. The survey asked stakeholders to indicate whether a fact or key message was necessary to make an informed decision about CRC surveillance by selecting agree, neutral, or disagree (online supplementary file). All survey data were collected anonymously, and we did not collect demographic information from the stakeholder survey participants. Analysis of the stakeholder surveys included counts and proportions.

\section{RESULTS}

\section{Description of patients and stakeholders}

A total of 67 patients were contacted and 31 patients (46\% response rate) were interviewed from three different medical centres (table 2). One patient was not included in the analysis because of a diagnosis of Lynch 
Table 1 Patientknowledge coding framework

\begin{tabular}{|c|c|}
\hline Code & Definition \\
\hline Stage of diagnosis & $\begin{array}{l}\text { No: Does not accurately state the stage of diagnosis. } \\
\text { Yes: Able to accurately state the stage of diagnosis. }\end{array}$ \\
\hline Site of diagnosis & $\begin{array}{l}\text { No: Does not accurately state the site of diagnosis. } \\
\text { Yes: Able to accurately state the site of diagnosis. }\end{array}$ \\
\hline $\begin{array}{l}\text { How the cancer was } \\
\text { detected }\end{array}$ & $\begin{array}{l}\text { No: Unable to express how his or her cancer was detected. } \\
\text { Yes: Able to express how his or her cancer was detected. }\end{array}$ \\
\hline $\begin{array}{l}\text { Treatment modality and } \\
\text { sequence }\end{array}$ & $\begin{array}{l}\text { Low: Does not accurately describe or provide any details about how the cancer was detected. } \\
\text { Medium: Provides more detail regarding treatments such as sequence and type of surgery or } \\
\text { doses of chemotherapy. } \\
\text { High: Provides correct facts about modality and sequence, uses the correct terms for treatment } \\
\text { regimen (eg, can name the chemotherapy). }\end{array}$ \\
\hline $\begin{array}{l}\text { Surveillance tests } \\
\text { (eg, CEA, endoscopy, } \\
\text { colonoscopy, imaging) }\end{array}$ & $\begin{array}{l}\text { Low: Does not name the tests or names one test. } \\
\text { Medium: Can list some tests (2/4). } \\
\text { High: Knows most of the tests (3/4) or why they were being done. }\end{array}$ \\
\hline $\begin{array}{l}\text { Harms of surveillance } \\
\text { testing }\end{array}$ & $\begin{array}{l}\text { Low: Is not able to list any harms of testing. } \\
\text { Medium: Knows that risks exist but cannot explain or has poor understanding of the implications. } \\
\text { High: Has realistic understanding/quantification of harms (eg, radiation exposure secondary } \\
\text { cancer risk, but it is very low; false positives as a risk of over-surveillance). }\end{array}$ \\
\hline $\begin{array}{l}\text { Frequency of } \\
\text { surveillance tests }\end{array}$ & $\begin{array}{l}\text { Low: Has no idea. } \\
\text { Medium: Has some idea of testing frequency but is not communicated clearly/correctly. } \\
\text { High: Differentiates the different timing between the tests. }\end{array}$ \\
\hline Duration of follow-up & $\begin{array}{l}\text { Low: Has no idea. } \\
\text { Medium: Has some concept of duration. } \\
\text { High: Understands and can communicate duration; makes reference to appropriate timeline (eg, } 5 \\
\text { years). }\end{array}$ \\
\hline Purpose of surveillance & $\begin{array}{l}\text { Low: Unable to express the rationale for testing. } \\
\text { Medium: Able to express to make sure cancer is not coming back. } \\
\text { High: States that surveillance is to monitor for recurrence and toxicity for long-term effects, } \\
\text { references quality of life, or life planning. }\end{array}$ \\
\hline Site of recurrence & $\begin{array}{l}\text { Low: Believes that recurrence will be more likely to come back in the colon or has no idea where } \\
\text { recurrence will occur. } \\
\text { Medium: Believes that recurrence will occur somewhere other than the colon. } \\
\text { High: Differentiates between distant and local recurrence, and/or able to describe that recurrence } \\
\text { will likely occur in the liver or lungs. }\end{array}$ \\
\hline $\begin{array}{l}\text { Sense of risk for } \\
\text { recurrence }\end{array}$ & $\begin{array}{l}\text { Low: Cannot describe or unsure of his or her risk for recurrence. } \\
\text { Medium: Has a general sense of risk but lacks detail. } \\
\text { High: Appropriately characterises his or her risk (eg, risk of recurrence can be different for people; } \\
\text { distant is higher risk than local; earlier in post-treatment surveillance the risk is higher). }\end{array}$ \\
\hline $\begin{array}{l}\text { Natural history of } \\
\text { recurrence }\end{array}$ & $\begin{array}{l}\text { Low: Does not understand how recurrence develops. } \\
\text { Medium: Has a broad understanding of recurrence development. } \\
\text { High: Shows understanding of recurrence development. }\end{array}$ \\
\hline $\begin{array}{l}\text { Treatment options for } \\
\text { recurrence }\end{array}$ & $\begin{array}{l}\text { Low: Is unable to describe or believes treatment for recurrence will be easy and straightforward or } \\
\text { like what they had initially. } \\
\text { Medium: Mentions some options for treatment of recurrence and/or states that treatment will be } \\
\text { different from the treatment for their primary CRC. } \\
\text { High: Understands that treatment will be difficult. }\end{array}$ \\
\hline Likelihood of cure & $\begin{array}{l}\text { Low: Believes the likelihood of cure is high or has no idea. } \\
\text { Medium: Knows that it may be harder to cure but lacks complete understanding. } \\
\text { High: Knows that recurrence is very difficult to cure. }\end{array}$ \\
\hline
\end{tabular}

CEA, carcinoembrionic antigen; CRC, colorectal cancer.

Syndrome. More than half of the patients were male $(61 \%)$, and the majority were White $(71 \%)$, non-Hispanic $(90 \%)$ and married $(71 \%)$. There was a good representation of educational levels with slightly more than half of individuals with less than a college education $(52 \%)$. The majority of the patients were between 1 and 
Table 2 Patient characteristics $(n=31)$

\begin{tabular}{lc}
\hline & $\mathbf{n}(\%)$ \\
\hline Age (median, IQR) & $60(53-68)$ \\
\hline Gender & \\
\hline Male & $19(61.3)$ \\
\hline Female & $12(38.7)$ \\
\hline Race & \\
\hline White & $22(71.0)$ \\
\hline Black & $5(16.1)$ \\
\hline Other & $4(12.9)$ \\
\hline Ethnicity & \\
\hline Non-Hispanic & $28(90.3)$ \\
\hline Hispanic & $3(9.7)$ \\
\hline Education & \\
\hline High school or less & $10(32.3)$ \\
\hline Some college/vocational training & $6(19.4)$ \\
\hline College & $10(32.3)$ \\
\hline Advanced degree & $5(16.1)$ \\
\hline Marital status & \\
\hline Married & $22(71.0)$ \\
\hline Not married & $9(29.0)$ \\
\hline Time from surgery (months) & \\
\hline $6-12$ & $5(16.1)$ \\
\hline 13-36 & $20(64.5)$ \\
\hline 37-48 & $6(19.4)$ \\
\hline Tumour site & \\
\hline Relon & $21(67.7)$ \\
\hline Stage at diagnosis & $10(32.3)$ \\
\hline Stage II & $3(9.7)$ \\
\hline & $5(16.1)$ \\
\hline Stage III & \\
\hline
\end{tabular}

3 years post-treatment, had colon cancer and were diagnosed at stage III. The anonymous stakeholder survey was completed by 49 participants (38 clinicians and 11 patient advocates). The clinicians represented practising academic and community clinicians and the patient advocates represented individuals engaged in clinical cancer research.

\section{Patients' knowledge of surveillance}

An overarching theme from the in-depth interviews was that patients had significant knowledge gaps regarding treatment, surveillance and recurrence (table 3). The overarching themes were consistent regardless of education level, marital status, or time since treatment. Patients generally had an accurate understanding of their stage at diagnosis $(77 \%, 23 / 30)$ and site of cancer $(85 \%, 22 / 26)$ based on their personal experience. However, there were patients who were confused about their stage and didn't completely grasp the difference between cancer in the colon or rectum.

Interviewer: It was colorectal, so was it in your rectum or what-or was it?

Patient: Yeah, I guess. I guess you'd say that

Interviewer: Okay. Your colon?

Patient: They had me with a-you know-a ostomy-a colostomy bag (Patient 31)

Overall, despite a desire to monitor for recurrence, patients had an incomplete understanding of recurrence mechanism, site, natural history and potential for cure (table 3). Very few patients could define cancer recurrence or describe where and when recurrence was most likely to occur. They described recurrence as 'cancer coming back-Patient 03' and a minority of patients could state that recurrence was most likely to occur in the lungs or liver. Few understood the limited potential for cure after recurrence, which was demonstrated by the belief that the treatment for recurrence would be the same as the treatment for the primary cancer: "I think pretty much probably the same, chemo and surgery, maybe radiation-Patient 04". Many confused recurrence with a new primary colon cancer ("Because you can have cancer further up-Patient 05"). Patients commonly believed that if recurrence was found early, there was a higher likelihood for cure; this concept may have been influenced by broader concepts of CRC screening and early detection of primary disease. For instance, one patient's description of the purpose of surveillance demonstrates the inability to differentiate screening and early detection of primary disease and surveillance for detection of recurrence, which is compounded by the belief that recurrence of cancer can be prevented. "To make sure [...] that nothing has changed in the body [...] this time they found polyps [...] It's also preventative. - Patient 05".

Patients had little understanding of the concept of post-treatment surveillance (table 3 ). They were able to broadly list the tests involved and general frequency of these tests, but it was from their experience and not from a deeper understanding of the reasons for the tests or the rationale for testing frequency or duration. Additionally, patients did not seem to think there were specific harms to surveillance testing, or they mentioned harms in passing. For example, one patient mentioned radiation as a possible harm but of minimal concern. Concern for false positive test results and possible need for additional testing also did not arise.

Patients equated the term surveillance with 'follow-up', but in general lacked a granular understanding of the purpose of surveillance and its implications for survival. However, one patient differentiated between 'surveillance' and 'follow-up'. For this patient, 'surveillance' was clearly about detecting recurrence: "Okay. So, in my mind, surveillance is looking for a recurrence or a metastasis. Like the scans I consider surveillance. The CEA level I consider 
Table 3 Patient knowledge of post-treatment colorectal cancer surveillance and recurrence

\begin{tabular}{|c|c|c|c|c|}
\hline & & Quality o & essed knc & \\
\hline & $\begin{array}{l}\text { Mentioned } \\
\text { n (\%) }\end{array}$ & $\begin{array}{l}\text { Low } \\
\text { n (\%) }\end{array}$ & $\begin{array}{l}\text { Medium } \\
\text { n (\%) }\end{array}$ & $\begin{array}{l}\text { High } \\
\text { n (\%) }\end{array}$ \\
\hline Types of surveillance tests & $30(96.8)$ & $5(16.7)$ & $14(46.7)$ & $11(36.7)$ \\
\hline Frequency of surveillance tests & $31(100.0)$ & 8 (25.9) & $21(67.7)$ & $2(6.5)$ \\
\hline Purpose of surveillance & $31(100.0)$ & $5(16.1)$ & $20(64.5)$ & $6(19.4)$ \\
\hline Site of recurrence & $27(87.1)$ & $20(74.1)$ & $4(14.8)$ & $3(11.1)$ \\
\hline Sense of risk for recurrence & $24(77.4)$ & $16(66.7)$ & $5(20.1)$ & $3(12.5)$ \\
\hline Natural history of recurrence & $19(61.3)$ & $14(73.1)$ & $4(21.1)$ & $1(5.3)$ \\
\hline
\end{tabular}

${ }^{*}$ The quality of expressed knowledge is based on the denominator of those mentioning the knowledge element.

surveillance. The colonoscopy and the sigmoidoscopy I consider surveillance.-Patient 21 ".

The concept of 'follow-up' was about maintaining quality of life:

And then follow-up care-I mean since I had my ileostomy reversal in [date]-I mean I'm not sure how familiar you are with-you know-kind of how that goes and what the healing is like, but I feel like it was a really long haul and it's been almost more difficult than having chemo. It's kind of getting back to like regular bowel function. So, I was seeing-and this is where I've gotten my just kind of follow-up care that's not surveillance, but I had visits with a nutritionist I had visits with another nurse in my surgeon's practice. I had visits with a pelvic floor physical therapist. - Patient 21

Although some patients stated they were going to be followed for 5 years, they had little to no understanding of the rationale for the follow-up time window. For those who were not able to express the 5-year duration, they expressed a belief that they would always be followed. For example one patient said "I always expect to be-you knowchecked up on once in a while. You know? Just to make sure it didn't come back. - Patient 06'.

\section{Stakeholder opinions regarding information patients should know about CRC surveillance}

Advocates and clinicians agreed that slightly half of the topics (six out of 13 topics) were important for patients to know: duration and frequency of surveillance, tests used for surveillance, the purpose of surveillance, timing of recurrence, definition of recurrence and basic CRC facts (figure 1). There was disagreement on four of these topics.

More advocates agreed that patients should know site of recurrence (90\% of advocates vs $65 \%$ of clinicians), the treatment options for recurrence $(100 \%$ of advocates vs $58 \%$ of clinicians), goals of treatment (eg, curative or palliative) for recurrence (100\% of advocates vs $38 \%$ clinicians), and potential harms of surveillance testing $(100 \%$ vs $68 \%)$ compared with clinicians. Compared with clinicians, somewhat fewer advocates agreed that patients should know the impact of surveillance on survival $(55 \%$ of advocates vs $70 \%$ of clinicians) and situations where surveillance may not be beneficial, such as advanced age ( $55 \%$ of advocates vs $76 \%$ of clinicians).

\section{DISCUSSION}

This exploratory study highlights areas for consideration regarding patients' informational needs and stakeholders' opinions regarding what patients should understand about surveillance and recurrence in order to make informed choices for their care. The in-depth interviews suggested that patients understood their diagnosis and treatment, but had significant knowledge gaps regarding recurrence and the purpose of post-treatment surveillance. The stakeholder survey suggested that advocates and clinicians differed in their opinions of what patients should know about surveillance and recurrence.

Patients' misperceptions about surveillance and recurrence is an important barrier for active participation in their care. ${ }^{10}$ The in-depth interviews suggested that patients do not have sufficient knowledge to actively participate in their post-treatment care; however, they did have a broad understanding of their diagnosis and treatment. Salz and colleagues found that most survivors of CRC remembered information about their treatment, but had a poor grasp on their risk of local recurrence, distant recurrence, or developing a new primary CRC. ${ }^{8}$ In our study, we found that patients did not understand the purpose of the different surveillance tests, the underlying 


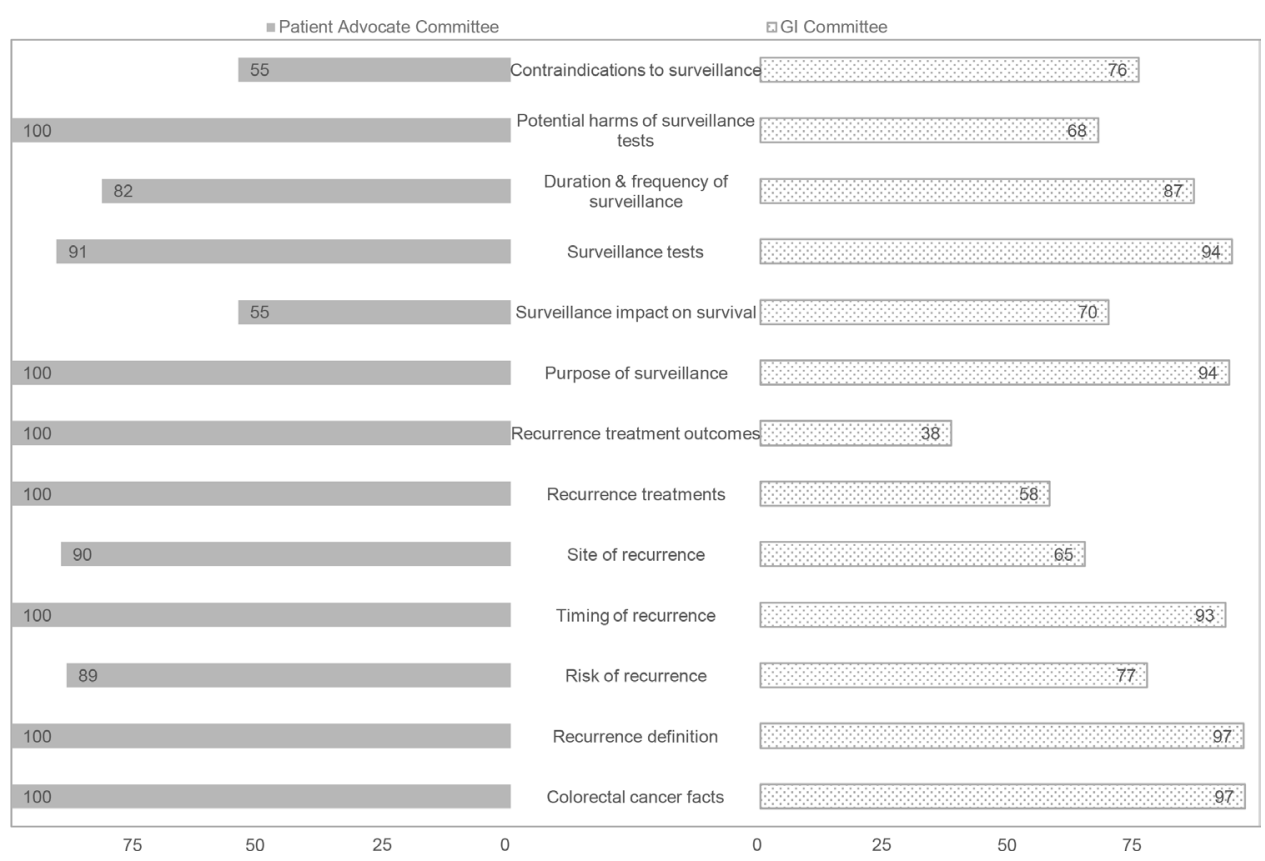

Figure 1 Comparing stakeholder responses this figure presents the stakeholder responses to what they feel patients should know about surveillance following curative resection of their colon or rectum. The values presented are in percentages. Gl, gastrointestinal.

rationale for the different timing of tests, the duration of surveillance, the natural history of recurrence and the likelihood of cure for recurrence. Our findings are similar to those of a study conducted with African American survivors of CRC, which revealed poor understanding of post-treatment surveillance testing and uncertainty about when they would be considered cured or no longer at significant risk for recurrence. ${ }^{11}$ The findings from this exploratory study suggests that these knowledge gaps are present regardless of education level, marital status, and time since treatment. Thus, clinicians need effective strategies to better educate patients about CRC surveillance.

Patients' misunderstanding of CRC surveillance could be problematic for clinicians as well. Fear of recurrence is one of the most important concerns among survivors of cancer. ${ }^{12}$ They can experience significant anxiety about the risk for recurrence, which can be out of proportion to their actual risk and look to their clinicians to alleviate this anxiety, often with the expectation of evaluating their cancer status with a test. Clinicians may have a difficult time explaining to patients the indications and limitations of testing, the appropriate frequency, or why their visits are becoming less frequent and will eventually end after 5 years, especially for patients who have a high fear of recurrence and are hesitant to separate from their oncology clinicians. ${ }^{13}$ In the event of recurrence, clinicians will have to explain that treatment for recurrence is likely to be more difficult. However, this reality could influence patients' underlying desire for testing. These very difficult and potentially time-consuming conversations require communication of difficult concepts with careful attention to use of plain language. ${ }^{14}$
From our stakeholder surveys, there was disagreement between advocates and clinicians regarding what facts or key messages need to be discussed. Our results confirm prior reports of this disconnect and highlight the importance of patient-centred care ${ }^{131516}$ Since not all patients who are identified with cancer recurrence will be eligible for curative-intent treatment, the role of intensive surveillance testing in such patients can be debated. However, compared with clinicians, fewer advocates felt that patients should know about contraindications to surveillance, or the potential for limited impact of surveillance on survival. These findings may reflect a belief that everyone has the right to receipt of care, even when treatment may not be beneficial, and that patients must continue to fight cancer by monitoring its recurrence. The data may also underpin patients' unwillingness to consider situations of medical futility in patients who might be too frail to undergo salvage surgery. Far fewer clinicians agreed that patients should know the potential harms of surveillance tests, recurrence treatment outcomes, potential treatments for recurrence, sites where recurrence could occur, and risk of recurrence. These results may suggest that clinicians are hesitant to get into specifics about recurrence, preferring to focus on the patient being 'cancer free' for now. This idea is consistent with the results of a study analysing patients and clinicians for post-treatment surveillance of pancreatic cancer, a disease where few options exist for treatment should recurrence be identified. ${ }^{13}$ It could also reflect clinicians' difficulty with providing individualised information on prognosis while still providing hope. $^{1718}$ 
A limitation of this study is the small sample size; however, thematic saturation was reached within 10-15 interviews regarding patients' expressed knowledge and new thematic insights are unlikely to be achieved simply by interviewing more patients. The sample was also diverse with respect to education level and included patients from a safety-net hospital. Another potential limitation is the effect of the interviewer on the participants' responses. Strategies were implemented to minimise the impact of the interviewers on the participants, such as, asking open ended questions, avoiding leading questions and not offering an opinion when queried. The generalisability of the results from the stakeholder survey is limited because the clinicians and advocates are highly engaged in research as part of their involvement in the National Cancer Institute Community Oncology Research Program. The advocates may not represent patients and caregivers at large as they are more engaged and knowledgeable about post-treatment surveillance for cancer. Since the survey was anonymous, we did not collect any additional demographic information from the clinicians nor patient advocates. The response rate could not be determined because the anonymous survey was distributed to attendees to the Gastrointestinal Committee and Patient Advocate Committee meetings which included individuals who may not have been clinicians nor patient advocates.

In summary, the findings from this exploratory study suggests patients have a significant knowledge gap regarding post-treatment surveillance and recurrence. There is a strong belief among advocates that clinicians should attempt to help their patients to be more informed about their disease and associated treatments. Patients need educational interventions to address these gaps to be more active in their care. A prior study found that survivors of cancer were unsatisfied with the available cancer information and that the need for cancer information decreased over time, but only among women. ${ }^{19}$ This latter study included individuals who were beyond the 5-year post-treatment surveillance period; thereby, limiting this findings relevance for patients during the post-treatment surveillance period. A more recent study with patients found that the need for information declined over time. ${ }^{20}$ Clinicians would also benefit from interventions to promote conversations about post-treatment surveillance of CRC that more closely align with the information needs of patients. One promising approach is educational interventions combined with communication skills training for clinicians. The communication skills training could focus on scaffolding clinicians' ability to share information that is responsive to each patients' desire and need for information, for example, how much. This approach recognises that patients differ in the amount of information they want. For instance, some patients want as much information as possible and others may be overwhelmed by too much information. ${ }^{21}$ Such interventions could help patients and clinicians be clear about the goals of care during post-treatment surveillance and recurrence, resulting in more patient-centred care.

\section{Author affiliations}

${ }^{1}$ Department of Health Services Research, The University of Texas MD Anderson Cancer Center, Houston, Texas, USA

${ }^{2}$ Department of Surgical Oncology, The University of Texas MD Anderson Cancer Center, Houston, Texas, USA

${ }^{3}$ Department of Medicine, University of California San Francisco, San Francisco, California, USA

${ }^{4}$ Department of Surgery, The University of Texas Health Science Center, Houston, Texas, USA

${ }^{5}$ Dana-Farber/Partners Cancer Care, Boston, Massachusetts, USA

${ }^{6}$ Patient Advocate, Marne, lowa, USA

Acknowledgements The authors thank Vincent $F$ Richards for his assistance with data collection, Dr Gary Deyter for his careful review, and Dr Lillian S Kao for assisting with providing access to patients for the interviews. This work was sponsored in part by the Cancer Care Delivery Research Committee of the Alliance for Clinical Trials in Oncology Network Research Base and the NCl Community Oncology Research Program, with special acknowledgment to the members of the Alliance Patient Advocates and the Gastrointestinal Cancers committees for their participation in the stakeholder survey.

Contributors LML contributed to the conceptualisation and design, collection and assembly of data, data analysis and interpretation, manuscript writing, and final approval of manuscript. RJV contributed to the conceptualisation and design, data interpretation, manuscript writing, and final approval of manuscript. AC contributed to the collection of data, data analysis and interpretation, manuscript writing, and final approval. APH contributed to data collection and interpretation, manuscript editing, and final approval of manuscript. YNY, KVL, SM, JAM and PG contributed to data interpretation, manuscript writing, and final approval of manuscript. GJC contributed to the conceptualisation and design, data analysis and interpretation, manuscript writing, and final approval of manuscript.

Funding Research reported in this publication was supported by the PatientCentered Outcomes Research Institute (PCORI) Award [CE13-04-6855, GJC]. This work was also supported, in part, by a grant from The University of Texas MD Anderson Cancer Center Duncan Family Institute for Cancer Prevention and Risk Assessment and by the National Institutes of Health through the MD Anderson Cancer Center Support Grant (P30CA016672). All statements in this publication, including, its findings, are solely those of the authors and do not necessarily represent the views of the Patient-Centered Outcomes Research Institute (PCORI), its Board of Governors or Methodology Committee. The content is solely the responsibility of the authors and does not necessarily represent the official views of the National Institutes of Health.

Competing interests None declared.

Patient consent for publication Not required.

Ethics approval This study was approved by The University of Texas MD Anderson Cancer Center Institutional Review Board (Interview IRB \#: PA13-1002, Stakeholder Survey IRB \# PA14-0935).

Provenance and peer review Not commissioned; externally peer reviewed. Data availability statement Data are available upon reasonable request.

Open access This is an open access article distributed in accordance with the Creative Commons Attribution Non Commercial (CC BY-NC 4.0) license, which permits others to distribute, remix, adapt, build upon this work non-commercially, and license their derivative works on different terms, provided the original work is properly cited, appropriate credit is given, any changes made indicated, and the use is non-commercial. See: http://creativecommons.org/licenses/by-nc/4.0/.

\section{REFERENCES}

1. Miller KD, Siegel RL, Lin CC, et al. Cancer treatment and survivorship statistics, 2016. CA Cancer J Clin 2016;66:271-89.

2. Siegel RL, Miller KD, Jemal A. Cancer statistics, 2016. CA Cancer J Clin 2016;66:7-30.

3. Kjeldsen BJ, Kronborg O, Fenger C, et al. The pattern of recurrent colorectal cancer in a prospective randomised study 
and the characteristics of diagnostic tests. Int $\mathrm{J}$ Colorectal Dis 1997;12:329-34.

4. Gill S, Loprinzi CL, Sargent DJ, et al. Pooled analysis of fluorouracilbased adjuvant therapy for stage II and III colon cancer: who benefits and by how much? J Clin Oncol 2004;22:1797-806.

5. Goldberg RM, Fleming TR, Tangen CM, et al. Surgery for recurrent colon cancer: strategies for identifying resectable recurrence and success rates after resection. eastern cooperative Oncology group, the North central cancer treatment group, and the southwest Oncology Group. Ann Intern Med 1998;129:27-35.

6. Primrose JN, Perera R, Gray A, et al. Effect of 3 to 5 years of scheduled CEA and CT follow-up to detect recurrence of colorectal cancer: the FACS randomized clinical trial. JAMA 2014;311:263-70.

7. Baca B, Beart RW, Etzioni DA. Surveillance after colorectal cancer resection: a systematic review. Dis Colon Rectum 2011;54:1036-48.

8. Salz T, Baxi SS, Blinder VS, et al. Colorectal cancer survivors' needs and preferences for survivorship information. $J$ Oncol Pract 2014;10:e277-82.

9. Gale NK, Heath G, Cameron E, et al. Using the framework method for the analysis of qualitative data in multi-disciplinary health research. BMC Med Res Methodol 2013;13:117.

10. Spatz ES, Krumholz HM, Moulton BW. The new era of informed consent: getting to a reasonable-patient standard through shared decision making. JAMA 2016;315:2063-4.

11. Pisu M, Holt CL, Brown-Galvan A, et al. Surveillance instructions and knowledge among African American colorectal cancer survivors. $J$ Oncol Pract 2014;10:e45-50.

12. Baker F, Denniston M, Smith T, et al. Adult cancer survivors: how are they faring? Cancer 2005;104(11 Suppl):2565-76.
13. Deobald RG, Cheng ESW, Ko Y-J, et al. A qualitative study of patient and clinician attitudes regarding surveillance after a resection of pancreatic and peri-ampullary cancer. HPB 2015;17:409-15.

14. Agency for Healthcare Research and Quality, Rockville, MD. Plain language at AHRQ. Available: http:www.ahrq.gov/policy/electronic/ plain-writing/index.html [Accessed 26 Dec 2016].

15. Butow P, Venkatesvaren L, Tattersall M, et al. Older patients preferences for prognostic information and involvement in decisionmaking: concordance between patients and doctors. PsychoOncology 2011;20:5-6.

16. IOM (Institute of Medicine). Delivering high-quality cancer care: charting a new course for a system in crisis. Washington, DC: The National Academies Press, 2013.

17. Hagerty RG, Butow PN, Ellis PM, et al. Communicating prognosis in cancer care: a systematic review of the literature. Ann Oncol 2005;16:1005-53.

18. Elit L, Charles C, Gafni A, et al. Walking a tightrope: oncologists' perspective on providing information to women with recurrent ovarian cancer (ROC) during the medical encounter. Support Care Cancer 2012;20:2327-33.

19. Mclnnes DK, Cleary PD, Stein KD, et al. Perceptions of cancerrelated information among cancer survivors: a report from the American cancer Society's studies of cancer survivors. Cancer 2008;113:1471-9.

20. Tan ASL, Nagler RH, Hornik RC, et al. Evolving information needs among colon, breast, and prostate cancer survivors: results from a longitudinal mixed-effects analysis. Cancer Epidemiol Biomarkers Prev 2015;24:1071-8.

21. Brütting J, Bergmann M, Garzarolli M, et al. Unmet information needs of patients with melanoma in Germany. Melanoma Res 2019;29:196-204. 